\title{
On the location and composition of the dust in the MCG-6-30-15 warm absorber
}

\author{
D. R. Ballantyne, J. C. Weingartner, and N. Murray ${ }^{\star}$
}

\author{
Canadian Institute for Theoretical Astrophysics, McLennan Labs, 60 St. George Street, Toronto, Ontario M5S 3H8, Canada \\ e-mail: ballantyne, weingart, murray@cita.utoronto.ca
}

Received 26 May 2003 / Accepted 23 July 2003

\begin{abstract}
The warm absorber observed in the Seyfert 1 galaxy MCG-6-30-15 is known to consist of at least two zones and very likely contains dust. Hubble Space Telescope images of MCG-6-30-15 show a dust lane crossing the galaxy just below the nucleus. In this paper, we argue that this dust lane is responsible for the observed reddening of the nuclear emission and the Fe I edge hinted at in the Chandra spectrum of MCG-6-30-15. We further suggest that the gas within the dust lane can comprise much of the low ionization component (i.e., the one contributing the O VII edge) of the observed warm absorber. Moreover, placing the warm absorbing material at such distances (hundreds of pc) can account for the small outflow velocities of the low ionization absorption lines as well as the constancy of the O VII edge. Photoionization models of a dusty interstellar gas cloud (with a column appropriate for the reddening toward MCG-6-30-15) using a toy Seyfert 1 spectral energy distribution show that it is possible to obtain a significant $\mathrm{O}$ VII edge $(\tau \sim 0.2)$ if the material is $\sim 150 \mathrm{pc}$ from the ionizing source. For MCG-6-30-15, such a distance is consistent with the observed dust lane. We emphasize the point first made by Kraemer et al.: dusty interstellar material will likely contribute to the warm absorber, and should be included in spectral modeling.

The current data on MCG-6-30-15 is unable to constrain the dust composition within the warm absorber. Astronomical silicate is a viable candidate, but there are indications of a very low $\mathrm{O}$ abundance in the dust, which is inconsistent with a silicate origin. If true, this may indicate that there were repeated cycles of grain destruction and growth from shocks in the interstellar medium of MCG-6-30-15. Pure iron grains are an unlikely dust constituent due to the limit on their abundance in the Galaxy, yet they cannot be ruled out. The high column densities inferred from the highly ionized zone of the warm absorber implies that this gas is dust-free.
\end{abstract}

Key words. dust, extinction - galaxies: active - galaxies: Seyfert - galaxies: individual: MCG-6-30-15 - X-rays: galaxies X-rays: ISM

\section{Introduction}

Soft X-ray absorption by photoionized gas was first used by Halpern (1984) and Pan et al. (1990) to explain the unusual spectrum of the quasar MR 2251-178. This "warm absorber" was characterized by ROSAT spectra of Seyfert $1 \mathrm{~s}$ which showed absorption edges due to O VII and O VIII (Nandra \& Pounds 1992; Nandra et al. 1993; Fiore et al. 1993; Turner et al. 1993). Warm absorber studies matured with the launch of the more sensitive ASCA observatory, which allowed more detailed spectral modeling (e.g., Fabian et al. 1994; George et al. 1995; Reynolds \& Fabian 1995). Analysis of a large sample of Seyfert 1s observed by ASCA showed that approximately $50 \%$ exhibit absorption features from photoionized gas in their soft X-ray spectra (Reynolds 1997; George et al. 1998).

\footnotetext{
Send offprint requests to: D. Ballantyne,

e-mail: ballantyne@cita.utoronto.ca

* Canada Research Chair in Astrophysics.
}

Currently, the study of the warm absorber is being revolutionized with observations from the dispersion gratings onboard Chandra and XMM-Newton which have been able to resolve individual absorption lines from a myriad of metals and charge states in the warm gas (e.g., Kaastra et al. 2000; Kaspi et al. 2001; Collinge et al. 2001; Lee et al. 2001; Kaspi et al. 2002). Photoionization modeling has then shown that, in many cases, more than one ionization parameter ${ }^{1}$ is needed to describe the observed spectrum (e.g, Morales et al. 2000; Kaspi et al. 2001; Kaastra et al. 2002). Also, the positions of the lines point toward outflow velocities on the order of a few hundred to a few thousand $\mathrm{km} \mathrm{s}^{-1}$. Thus, this warm absorbing gas seems to be in the form of an outflowing wind (cf., Bottorff et al. 2000; Elvis 2000). However, there remains a major uncertainty in the

\footnotetext{
1 There are some objects which can still be modeled adequately with only one ionization parameter, e.g., Mrk 509 (Yaqoob et al. 2003).
} 
location of the gas, with many models considering the broadline region (e.g., Reynolds \& Fabian 1995; Netzer 1996; George et al. 1998) or the putative obscuring torus (e.g., Krolik \& Kriss 1995,2001$)$ as the most likely origin for the warm absorber.

A possible constraint on the location of at least some of this warm gas may be provided if it contains any dust, which sublimates at the radius of the broad-line region (Barvainis 1987) for a typical active galactic nucleus (AGN). Dusty warm absorbers (hereafter, DWA) were first considered for the quasar IRAS 13349+2438 (Brandt et al. 1996; Siebert et al. 1999) and the Seyfert 1 galaxy MCG-6-30-15 (Reynolds et al. 1997). In both of these AGN, the column of neutral $\mathrm{H}$ inferred from the reddening is significantly larger than that inferred from the neutral absorption in the soft X-ray band, but is of the same order as the column of ionized gas inferred from the warm absorber (Reynolds et al. 1997). This suggests that the dust is similar to Galactic dust and resides within the warm ionized gas, and may significantly affect the observed soft X-ray spectrum (Komossa \& Fink 1997 and references therein; Komossa \& Bade 1998). Spectroscopic evidence for dust in X-ray warm absorbers has now been found by Lee et al. (2001) in the Chandra gratings observation of MCG-6-30-15 (this seems to have been confirmed by the very recent XMM-Newton data of Turner et al. 2003). This high resolution spectrum exhibited a sharp drop ${ }^{2}$ at $\sim 0.7 \mathrm{keV}$ consistent with the L3 absorption edge from neutral $\mathrm{Fe}$. A similar feature was also found in a Chandra spectrum of the Galactic X-ray binary Cyg X-1 (Schulz et al. 2002). The column implied by the depth of the Fe edge in MCG-6-30-15 is of the right order to explain the observed reddening $\left(A_{V} \approx \tau_{V} \approx 3 E(B-V)=1.8\right.$; Reynolds et al. 1997), assuming Galactic-type dust located within the warm absorber.

Rather than place the DWA near the central engine of the AGN, Kraemer et al. (2000) (see also Crenshaw \& Kraemer 2001) argued for the existence of a "lukewarm absorber" outside the narrow-line region. This gas would have sufficient column to explain the observed reddening, and has been ionized to the point where hydrogen is fully stripped, but the metals would only be moderately ionized and would exhibit strong UV absorption lines rather than O VII or O VIII edges. Thus, this model requires an inner warm absorber to account for the highly ionized oxygen features (Kraemer et al. 2000). The lukewarm absorber has been shown to be consistent with the X-ray (Kraemer et al. 2000) and UV (Crenshaw et al. 2001) data of NGC 3227, as well as the UV spectrum of Ark 564 (Crenshaw et al. 2002).

In the case of MCG-6-30-15 $\left(z=0.008, L_{2-10 \mathrm{keV}} \approx\right.$ $10^{43} \mathrm{erg} \mathrm{s}^{-1}$; see Table 1 for a summary of the absorbing columns), it was clear from early $A S C A$ variability studies that a multi-zone warm absorber was needed (e.g., Otani et al. 1996; Morales et al. 2000). In particular, the O VIII edge was found

\footnotetext{
${ }^{2}$ An alternative explanation for this drop (based on XMM-Newton data) is that it is the blue edge of a relativistically broadened O VIII Ly $\alpha$ line (Branduardi-Raymont et al. 2001; Sako et al. 2003). However, this interpretation has been challenged from both observational (Lee et al. 2001) and theoretical (Ballantyne et al. 2002) points of view.
}

Table 1. A summary of the absorbing columns toward MCG-6-30-15. Reference 1 = Elvis et al. (1989), 2 = Reynolds et al. (1997), 3 = Lee et al. (2002).

\begin{tabular}{ccccc}
\hline \hline Type & $N_{\mathrm{H}}\left(\mathrm{cm}^{-2}\right)$ & Derived from & Origin & Ref. \\
\hline Cold & $4.06 \times 10^{20}$ & soft X-ray cutoff & Galaxy & 1 \\
?? & $4 \times 10^{21}$ & reddening & intrinsic & 2 \\
Warm & $\sim 10^{23}$ & soft X-ray edges and lines & intrinsic & 3 \\
\hline
\end{tabular}

Fig. 1. Hubble Space Telescope image of MCG-6-30-15 (Malkan et al. 1998). A dust lane is apparent crossing the southern part of the galactic disk.

to anticorrelate with the source luminosity while the O VII edge seemed to remain constant (Otani et al. 1996; Orr et al. 1997). The Chandra observations found a strong O VII edge $\left(\tau_{\text {OVII }} \sim 0.7\right)$ and a series of $\mathrm{O}$ VII absorption lines within $200 \mathrm{~km} \mathrm{~s}^{-1}$ of the systemic velocity of MCG-6-30-15 (Lee et al. 2001). An updated analysis presented by Lee et al. (2002) showed that many of the other low-ionization absorption lines also have very small outflow velocities. These data point to a distribution (in space/velocity) of ionized absorbers, consistent with the earlier ASCA results.

A Hubble Space Telescope (HST) image of MCG-6-30-15 shows a distinct dust lane that cuts across the southern part of the galactic disk (Fig. 1; Malkan et al. 1998; see also Ferruit et al. 2000). This material is likely intrinsic to the galaxy and not a foreground effect such as Galactic cirrus (e.g., M81; Abolins \& Rice 1987). Therefore, in Sect. 2, we follow the ideas of Kraemer et al. (2000), but argue that at least part of the low ionization warm absorber and all of the observed reddening in MCG-6-30-15 originates within this dust lane. It is not our intention to construct a detailed spectral model of the DWA in MCG-6-30-15; rather we use this system as an example of how material in the dusty interstellar medium (ISM) of the host galaxy can have a significant observational impact on the study of absorption in AGN spectra.

The composition of the dust in the MCG-6-30-15 DWA is also a puzzle. For Cyg X-1, where the high signal-to-noise allowed a comparison to laboratory data, Schulz et al. (2002) 
claimed that pure metallic Fe was the best fit to the edge structure. This is surprising, since the expected microwave emission (at $\approx 90 \mathrm{GHz}$ ) from such grains is not observed in the Galaxy (Draine \& Lazarian 1999). Lee et al. (2001) argued that the Cyg X-1 iron edge was similar to the one observed in MCG-6-30-15, and thus pure iron grains may be present in the DWA. In Sect. 3 we discuss the constraints on a pure iron component in astronomical dust, and consider if it is a plausible composition for the dust in the MCG-6-30-15 DWA.

\section{The location of the DWA}

The dust lane seen in Fig. 1 passes just south of the nucleus, so, judging from the Galactic distribution (Dickey \& Lockman 1990), our line of sight likely passes through a column of a few times $10^{21} \mathrm{~cm}^{-2}$. This is of the same order as that needed to provide the observed reddening in MCG-6-30-15 assuming Galactic-type grains $\left(N_{\mathrm{H}} \approx 4 \times 10^{21} \mathrm{~cm}^{-2}\right.$; Reynolds et al. 1997). Further evidence for a low density origin of the DWA is the recombination timescale argument of Otani et al. (1996). They noted that the constancy of the O VII edge in the long 1994 ASCA observation of MCG-6-30-15 implies $n_{\mathrm{H}}<2 \times 10^{5} \mathrm{~cm}^{-3}$ in the outer absorber. ISM gas with a fixed hydrogen number density of $n_{\mathrm{H}}=10 \mathrm{~cm}^{-3}$ will be used as the canonical absorber for the remainder of the paper.

A simple photoionization argument can be made to constrain the distance of the O VII absorber. We define the following ionization parameter

$U_{\mathrm{H}} \equiv \frac{L_{>}}{4 \pi r^{2} c n_{\mathrm{H}}\langle h v\rangle}$,

where $L_{>}$is the AGN luminosity beyond the Lyman edge, $n_{\mathrm{H}}$ is the total hydrogen number density, and $\langle h v\rangle$ is an average ionizing photon energy. Photoionization equilibrium gives $\xi_{\mathrm{H}}=n_{\mathrm{H}^{0}} / n_{\mathrm{H}}=\left(\alpha_{\mathrm{H}}(T) / a_{\mathrm{H}} c\right) U_{\mathrm{H}}^{-1}$, where $n_{\mathrm{H}^{0}}$ is the number density of neutral $\mathrm{H}, \alpha_{\mathrm{H}}$ is the recombination coefficient of $\mathrm{H}$ at temperature $T$ and $a_{\mathrm{H}}$ is the photoionization cross-section for $H$. This expression is valid only when $\xi \ll 1$. Generalizing for a hydrogenic ion of an element with atomic number $Z$ gives

$\xi_{Z}=\frac{\alpha_{\mathrm{H}}(T)}{a_{\mathrm{H}} c} U_{\mathrm{H}}^{-1} Z^{4+2 \beta}=\left(1.4 \times 10^{-6}\right) U_{\mathrm{H}}^{-1} Z^{4+2 \beta}$,

where we have assumed $L_{v} \propto v^{-\beta}$ for the ionizing luminosity ( $\beta \approx 1.6$ for radio-quiet quasars; Telfer et al. 2002), and used $a_{\mathrm{H}}=6 \times 10^{-18} \mathrm{~cm}^{2}$ and $\alpha_{\mathrm{H}}(20000 \mathrm{~K})=2.5 \times 10^{-13} \mathrm{~cm}^{3} \mathrm{~s}^{-1}$ (Osterbrock 1989). With a typical Seyfert 1 Lyman luminosity of $L_{>}=10^{44} \mathrm{erg} \mathrm{s}^{-1}$, and assuming $\langle h v\rangle=45 \mathrm{eV}$, the ionization parameter of a $n_{\mathrm{H}}=10 \mathrm{~cm}^{-3}$ cloud at $1 \mathrm{kpc}$ is 0.04 . Equation (2) then gives $\xi_{\mathrm{H}} \approx 10^{-5}$ and $\xi_{\mathrm{O}} \approx 100$, implying that although hydrogen is still entirely ionized at this distance, oxygen has started to recombine. If we now consider gas only $10 \mathrm{pc}$ from the AGN, the values of $\xi$ will drop by $10^{4}$, and $\mathrm{O}$ is predominantly fully-stripped. Therefore, the $\mathrm{O}$ VII absorber must lie between 10 and $1000 \mathrm{pc}$. This calculation assumes photoionization equilibrium and is therefore valid only on timescales longer than the recombination time, $t_{\text {rec }} \sim 1 / n_{\mathrm{e}} \alpha_{\mathrm{H}}(T) \sim 10^{4}$ years, where $n_{\mathrm{e}}$ is the free electron density. The radial range derived above will be reasonable as long as the luminosity of the central engine did not change by factors greater than $\sim 10$ over $t_{\text {rec }}$. To more accurately determine the properties of an ISM warm absorber, numerical photoionization models must be employed.

We used Cloudy 96Beta4 (Ferland 2002) to predict the ionization structure of a $n_{\mathrm{H}}=10 \mathrm{~cm}^{-3}$ cloud of ISM gas at various distances from an AGN. The neutral hydrogen column density was fixed at $N_{\mathrm{H}}=4 \times 10^{21} \mathrm{~cm}^{-2}$, which, for a Galactic gasto-dust ratio, is the minimum needed to provide the reddening toward MCG-6-30-15 $\left(N_{\mathrm{H}}=4-7 \times 10^{21} \mathrm{~cm}^{-2}\right.$; Reynolds et al. 1997). The dust (silicate plus graphite) and gas-phase metal abundances were fixed at the ISM values described by Ferland (2002). The cloud was illuminated with a "standard" AGN continuum (see p. 34 in Ferland 2002) with $\alpha_{\text {ox }}=-1.4$ and a X-ray power-law photon-index $\Gamma=2$. The "big blue bump" was characterized by a temperature of $1.4 \times 10^{5} \mathrm{~K}$ (the maximum Shakura \& Sunyaev (1973) accretion disc temperature for a $10^{7} M_{\odot}$ black hole accreting at 0.1 of its Eddington rate) and a UV slope of $\alpha(\mathrm{UV})=-0.5$. This spectral energy distribution (SED) is not intended to be a realistic model of the MCG-6-30-15 continuum (which is unknown because of the large reddening), but rather representative of a generic AGN. The normalization of the SED was set by defining the 2$10 \mathrm{keV}$ luminosity to be $10^{43} \mathrm{erg} \mathrm{s}^{-1}$, typical of many Seyfert $1 \mathrm{~s}$ (Reynolds 1997).

Models were calculated with the inner edge of the gas cloud at various distances $r$ from the continuum source, and the computed $\mathrm{O}$ VII column density was compared with the result from the Chandra observation of MCG-6-30-15, $N_{\text {OVII }} \approx$ $2.5 \times 10^{18} \mathrm{~cm}^{-2}$ (Lee et al. 2001). The maximum O VII column found in the Cloudy runs was $6.1 \times 10^{17} \mathrm{~cm}^{-2}$ for a cloud distance of $150-175 \mathrm{pc}$. Models with the gas closer in were too ionized, and if the cloud was further out, it was not ionized enough. Of course, lowering the density by a factor $f$ would allow a distance $\sqrt{f}$ larger. However, if the distance exceeds $\approx 200-250 \mathrm{pc}$, then, even with $n_{\mathrm{H}}=10 \mathrm{~cm}^{-3}$, the physical length of the column exceeds the distance to the AGN. A similar calculation with a power-law SED of energy index -1.17 between 0.013 and $100 \mathrm{keV}$ resulted in $N_{\text {OVII }}=6.2 \times 10^{17} \mathrm{~cm}^{-2}$ at $r=150 \mathrm{pc}$. While these models cannot account for the entire O VII column in MCG-6-30-15 (it is likely that some fraction of the O VII edge originates within the inner warm absorber), we have shown that this dusty ISM cloud will have an detectable impact on the observed spectrum (see also Komossa \& Bade 1998).

To illustrate the extent of this impact, the incident and transmitted continua for the AGN models with $r=100,175$, and $250 \mathrm{pc}$ are shown in Fig. 2. These runs have $U_{\mathrm{H}}=3.7$, 1.2 and 0.6 , respectively. The transmitted spectra show significant warm absorption in the soft X-ray band with strong edges at $0.28,0.53$ and $0.74 \mathrm{keV}$. These first two are due to $\mathrm{C}$ I and $\mathrm{O}$ I in the grains (we include both graphite and silicate dust), while the latter is the O VII edge. Of the three models shown here, the depth of the O VII edge is greatest when $r=175 \mathrm{pc}$, with $\tau_{\text {OVII }}=0.15$. The Fe I edge from the dust is noticeable as a small notch just redward of the O VII edge, as was observed by Lee et al. (2001). The majority of the opacity below $0.3 \mathrm{keV}$ is due to absorption by He II, as was found in the lukewarm 


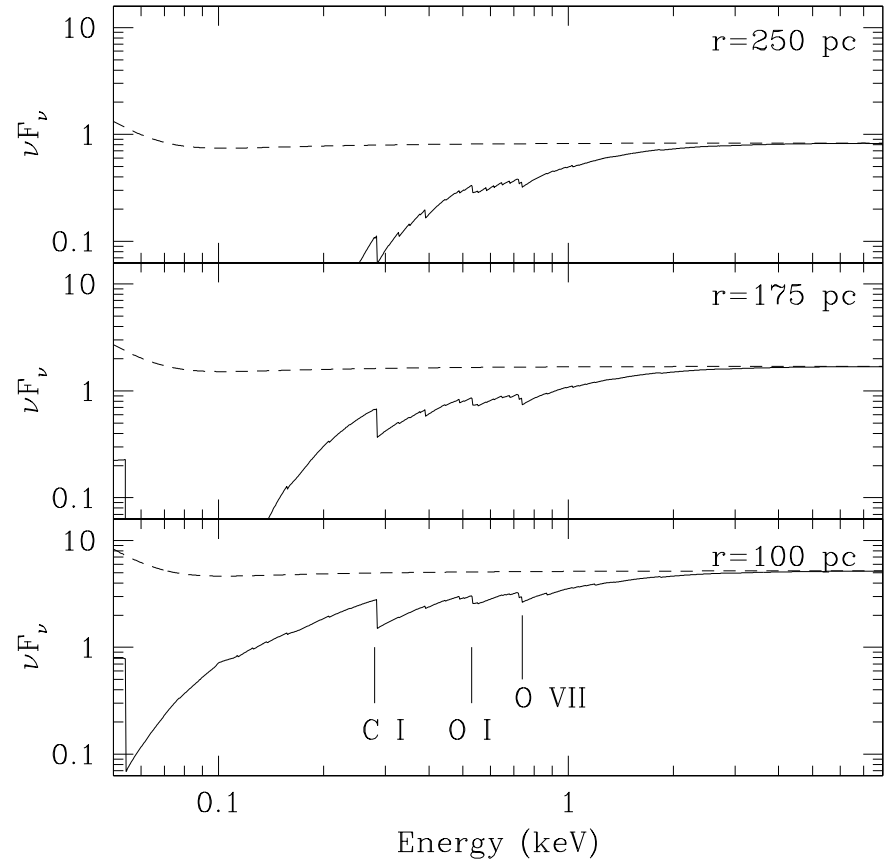

Fig. 2. The results of photoionization models of a DWA shown over the X-ray band. Constant density $\left(n_{\mathrm{H}}=10 \mathrm{~cm}^{-3}\right)$ gas with $N_{\mathrm{H}}=$ $4 \times 10^{21} \mathrm{~cm}^{-2}$ was illuminated by a toy AGN SED (shown with the dashed line), and the transmitted spectrum is shown with the solid line. Typical interstellar dust was included at Galactic abundances. The positions of the most prominent photoelectric absorption edges are indicated in the lower panel. The gas was placed at different distances $r$ from the AGN. The greatest column of O VII $\left(N_{\mathrm{OVII}}=6 \times 10^{17} \mathrm{~cm}^{-2}\right)$ was found when $r=150-175 \mathrm{pc}$.

absorber of Kraemer et al. (2000). Therefore, such absorption will need to be included in absorption models for sources such as MCG-6-30-15 where distant material is contributing to the warm absorber. Indeed, in their multi-zone DWA fit to the new XMM-Newton data, Turner et al. (2003) require a lowionization component to account for additional soft X-ray absorption that is inconsistent with cold absorption. It is quite likely that this excess attenuation is caused by He II absorption.

The fact that there is a significant column of He II $(5.9 \times$ $10^{18} \mathrm{~cm}^{-2}$ ) in the models shows that the gas is very close to recombining to a more neutral configuration. This is in large part due to the presence of dust which, for a Galactic size distribution, dominates the continuum opacity in the extreme UV (EUV) and soft X-ray bands (see Fig. 3). Indeed, our Cloudy models show that neutral oxygen begins to be present in the gas when $r=175 \mathrm{pc}$. Thus, our results seem to be fine-tuned in the sense that the greatest $\mathrm{O}$ VII column is found when the gas is on the verge of recombining. This argues that it may be difficult, in general, for ISM gas to contribute to the warm absorber.

In this section we have argued that ISM material associated with dust lanes in the host galaxy of an AGN is a natural contributor to the observed DWAs. Cloudy models suggest that this gas, when illuminated by a toy AGN SED, can produce significant warm absorption (including a significant $\mathrm{O}$ VII edge) when placed $\sim 150 \mathrm{pc}$ from the AGN. This may be particularly relevant to MCG-6-30-15, where a dust lane does pass just below the nucleus. Using Fig. 1 and the plate scale from

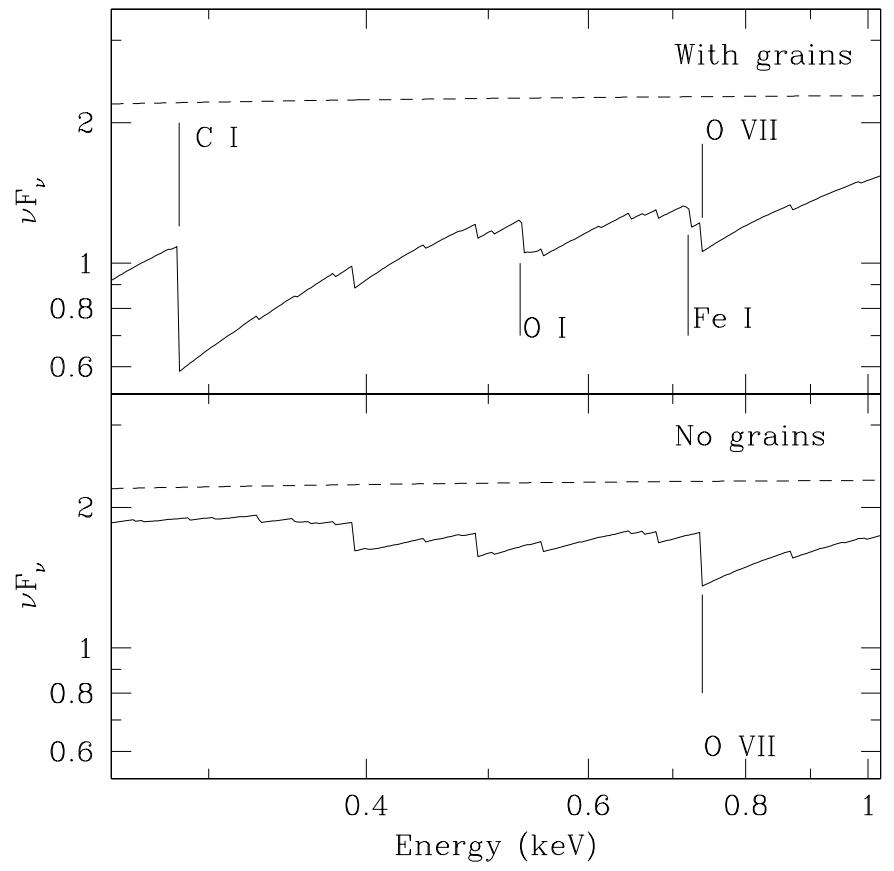

Fig. 3. The effect of grains on the transmitted spectrum (see also Komossa \& Bade 1998). Both panels show the results of Cloudy models where the gas cloud was $150 \mathrm{pc}$ from the AGN. The dashed curve shows the incident spectrum and the solid curve shows the transmitted spectrum. The positions of the C I, O I, Fe I, and O VII edges are indicated. Solar abundances were used for the dust-free model, except that the oxygen abundance was set to the F and G star value from Sofia \& Meyer (2001).

Malkan et al. (1998), we can estimate the inner edge of the dust lane from the HST image. Interestingly, this is also of the order of $150-450 \mathrm{pc}$, assuming $H_{0}=50 \mathrm{~km} \mathrm{~s}^{-1} \mathrm{Mpc}^{-1}$.

It may be difficult applying this idea to other sources which harbor DWAs (e.g., IRAS 13349+2438). The dust lane in MCG-6-30-15 lies nearly along our line of sight and may be a relatively unusual alignment. Nevertheless, the strong ionizing power of an AGN will have a great impact on its local ISM. The observational consequences of these effects will depend both on the viewing angle into the source (Crenshaw \& Kraemer 2001) and on the SED of the AGN. Indeed, if our lineof-sight into MCG-6-30-15 were slightly different and passed through the dust lane, rather than just above it, the obscuration could be high enough that the AGN would appear as a Seyfert 2 (see, e.g., Matt 2000).

\section{Dust properties}

\subsection{Composition}

As the EUV/soft X-ray opacity in the DWA is dominated by dust, the absorption properties depend on the grain composition. The top panel of Fig. 3 shows the transmitted spectrum for the $r=150 \mathrm{pc}$ model with Galactic silicate and graphite dust abundances (Mathis et al. 1977). In the bottom panel, the spectrum is displayed for the case where the elements in the dust have been returned to the gas. We assumed that the total 
(gas + dust) abundances are equal to solar (Grevesse \& Sauval 1998) except for $\mathrm{O}$, for which we took $\mathrm{O} / \mathrm{H}=4.45 \times 10^{-4}$, a value measured from local young $\mathrm{F}$ and $\mathrm{G}$ stars (Sofia \& Meyer 2001). This oxygen abundance has the advantage that it does not require depletions greater than what can be accounted for by dust. The DWA model exhibits a $\mathrm{C}$ I edge at $0.28 \mathrm{keV}$ of depth $\tau=0.62$ from the graphite dust, an $\mathrm{O}$ I edge at $0.53 \mathrm{keV}$ of depth $\tau=0.15$, and an Fe I edge at $0.72 \mathrm{keV}$ of depth $\tau=0.09$, both from the silicate dust. The dust-free model also shows absorption edges, the largest from O VII at $0.74 \mathrm{keV}$ of depth $\tau=0.26$.

Unfortunately, the High Energy Transmission Gratings (HETG) Chandra spectra only extend to an energy of $0.45 \mathrm{keV}$, so we have no X-ray evidence for or against the presence of graphite; however the Low Energy Transmission Gratings (LETG) can reach these low energies although the instrumental $\mathrm{C}$ edge will need to be well calibrated to measure the intrinsic edge due to graphite. The Reflection Grating Spectrometer (RGS) onboard XMM-Newton also does not go below $0.3 \mathrm{keV}$, although the low-resolution European Photon Imaging Cameras (EPIC) are able to do so.

As mentioned in Sect. 1, the strength of the measured Fe I edge from the Chandra observation of MCG-6-30-15 implies $N_{\mathrm{Fe} \text { I }} \sim 4 \times 10^{17} \mathrm{~cm}^{-2}$, consistent with the observed reddening for Galactic-type dust. If the Fe really is incorporated into silicate grains, then we expect $N_{\mathrm{OI}}>N_{\mathrm{Fe}}$, since a silicate structural unit contains more $\mathrm{O}$ atoms than $\mathrm{Fe}$ atoms. For example, the Cloudy models discussed above assume a $\mathrm{MgFeSiO}_{4}$ stoichiometry and predict an $\mathrm{O}$ I edge that is roughly the same depth as the Fe I edge. Lee et al. (2002) claimed that silicates with an O:Fe number ratio of $2: 1$ rather than $4: 1$ were consistent with the Chandra data, but also mentioned that the data were limited by statistics at the position of the $\mathrm{O} I$ edge. Therefore, if the silicate dust had an O:Fe ratio of $4: 1$, it could have been detected by Chandra. However, Sako et al. (2003) quote an upper limit of $N_{\mathrm{OI}}=10^{16} \mathrm{~cm}^{-2}$ from their XMM-Newton RGS observation of MCG-6-30-15, which has higher signal-to-noise, but lower resolution, than the Chandra spectrum. These authors also find $N_{\mathrm{FeI}}=7 \times 10^{16} \mathrm{~cm}^{-2}$ for the column of neutral iron, which implies a $V$-band optical depth of 0.07 (assuming a silicate grain radius of $0.1 \mu \mathrm{m}$ ). Therefore, this column could not account for the observed reddening of MCG-6-30-15, unless it is provided predominantly by ironpoor grains.

Further constraints on the mineralogical structure of the dust can, in principle, be provided by the detailed structure of the Fe I edge. Lee et al. (2001) found that the edge structure in MCG-6-30-15 matches very well that found in Cyg X-1. Schulz et al. (2002) showed that the Cyg X-1 edge could be fairly accurately reproduced if the $\mathrm{Fe}$ resides in pure $\mathrm{Fe}$ grains (taking experimental data from Kortright \& Kim 2000), but not if the $\mathrm{Fe}$ is in oxides (taking data from Crocombette et al. 1995). A measurement of the oxygen column in the dust could not be made for Cyg X-1, as the large O I edge $(\tau>2)$ was dominated by neutral oxygen in the ISM along the line-of-sight. Therefore, Schulz et al. (2002) concluded that pure iron grains were most consistent with the data, and Lee et al. (2001) present this as a possibility for the dust in
MCG-6-30-15. However, for Cyg X-1 metallic Fe dust is probably ruled out since it would produce excessive thermal magnetic dipole emission at $90 \mathrm{GHz}$ (Draine \& Lazarian 1999). Draine \& Lazarian suggest that at most $\sim 5 \%$ of the Galactic interstellar Fe can be in metallic iron grains. Thus, it seems unlikely that there is a significant column of metallic iron grains toward Cyg X-1. Although we have not performed a detailed spectral fitting, it appears that the observed edge structure in Cyg X-1 could be due to Fe in silicates, based on the spectrum of an olivine sample from Gloter et al. (2000).

The above argument cannot be directly applied to MCG-6-30-15 as there are no microwave observations of this galaxy, so the key to uncovering the dust composition in the DWA is an accurate measurement of other edges such as O I, $\mathrm{C}$ I, or Si I at $1.85 \mathrm{keV}\left(\tau_{\mathrm{Si}} \mathrm{I}<0.01\right.$ in the Cloudy models). The Chandra data may imply wüstite $(\mathrm{FeO})$ or hematite $\left(\alpha \mathrm{Fe}_{2} \mathrm{O}_{3}\right)$ dust, but the XMM-Newton limit by Sako et al. (2003) points to little or no oxygen in the grains.

Perhaps the lack of $\mathrm{O}$ I can be explained by selective destruction of O-bearing grains (e.g., silicates). Depletion patterns in the Galaxy suggest that $\mathrm{Fe}$ and $\mathrm{Si}$ are incorporated into different dust populations, with the Fe-bearing component significantly longer-lived than the Si-bearing component (e.g., Tielens 1998). If the dust in the DWA in MCG-6-30-15 were subjected to repeated episodes of destruction (in shocks) and re-growth, then the Si-bearing dust component could be largely removed while the Fe-bearing component remains largely intact. Such a process would lead to a reduced $E(B-V) / N_{\mathrm{H}}$. However, the metallicity at $r \sim 150 \mathrm{pc}$ from the nucleus of MCG-6-30-15 is probably higher than at the location of the Sun in the Galaxy, so that $E(B-V) / N_{\mathrm{H}}$ in the DWA in this scenario could be comparable to its value in the local ISM of the Galaxy. One potential difficulty for this scenario (and for silicate dust models in general) is that the destruction timescale inferred by Tielens (1998) for the Si-bearing dust is substantially shorter than predicted for silicate grains (Jones et al. 1996). Counter-intuitively, Jones et al. also find the lifetime of pure $\mathrm{Fe}$ dust to be shorter than the lifetimes of graphite or silicate dust, because Fe grains are accelerated to higher speeds in the shock. Weingartner \& Draine (1999) suggested the Fe may largely be incorporated into the carbonaceous dust population rather than the silicate component. In this picture, gas-phase $\mathrm{Fe}$ atoms rapidly accrete onto polycylic aromatic hydrocarbon molecules, forming organometallic "sandwich" molecules, which may ultimately coagulate to form larger grains. Laboratory Fe L-edge spectra of such compounds would be very useful.

\subsection{Other implications}

The Chandra data also place a constraint on the inner warm absorbing gas which is responsible for the O VIII edge in the ASCA data. From the depth of the high-ionization absorption lines, Lee et al. (2002) found $N_{\mathrm{H}} \sim 10^{23} \mathrm{~cm}^{-2}$ for this material. If the Fe I edge observation were not available one might argue that the inner absorber contains large grains that do not redden the optical nuclear emission. However, since all of the neutral 
Fe can be accounted for by the outer absorber, no such Febearing grains exist in the inner absorber, suggesting that the inner absorber is dust-free. This, in turn, suggests than the inner absorber is located within the dust sublimation radius, unless an alternative mechanism can be found for depleting the gas of dust at larger distances.

Indeed, if grains are to be found close to the central engine of an AGN, they must be shielded from the outpouring radiation. The radiation pressure opacity of dust is $\sim 10^{3}$ times that of electrons (Laor \& Draine 1993), so within a certain radius the dust will see a significantly super-Eddington source. The gravitational and radiation forces will balance when the dust particle is outside a mass $\sim 1000 M_{\mathrm{BH}}$, where $M_{\mathrm{BH}}$ is the black hole mass of the AGN. As an example, the inner velocity profiles of S0 galaxies are approximately linear (Seifert \& Scorza 1996; Loyer et al. 1998), implying that $M(r) \propto r^{3}$. Thus, if at $r=1 \mathrm{pc} M(r)=M_{\mathrm{BH}}$, then the balancing radius for the dust will be $\sim 10 \mathrm{pc}$, independent of the black hole mass. Therefore, if a DWA is at $150 \mathrm{pc}$ it will not feel a significant radiation force, and should not have a large outflow velocity. In the case of MCG-6-30-15, this is consistent with the preliminary results from the Chandra data (Lee et al. 2001, 2002), and the low velocity component inferred by Sako et al. (2003) from the XMM-Newton spectrum.

\section{Summary}

The DWA in MCG-6-30-15 has been the subject of two different X-ray gratings observations, but the interpretation of the spectra has been controversial (Branduardi-Raymont et al. 2001; Lee et al. 2001). However, the optical/UV reddening requires dust along the line-of-sight to the AGN. This dust will imprint observable $\mathrm{Fe}, \mathrm{C}, \mathrm{O}$ and $\mathrm{Si}$ edges on an X-ray spectrum, and indeed an Fe I edge was likely detected in the Chandra (Lee et al. 2001) and XMM-Newton (Turner et al. 2003) data. The gas associated with this dust has a column of a few times $10^{21} \mathrm{~cm}^{-2}$ and may be responsible for much of the lowionization component of the warm absorber in MCG-6-30-15 (that is, it produces the O VII edge).

Motivated by the the dust lane seen in the HST image of MCG-6-30-15, we have argued in this paper that DWAs may reside in the ISM of the host galaxy. Photoionization modeling shows that a detectable $\mathrm{O}$ VII edge can be produced by such material if it is placed $100-200 \mathrm{pc}$ away from the AGN and therefore can contribute to the warm absorption features. Furthermore, the dust contained within this gas is dynamically and thermally stable and can account for any observed reddening. Supporting evidence for this interpretation for MCG-6-30-15 is given by the very small outflow velocity of the low ionization absorption lines, the observed constancy of the $\mathrm{O}$ VII edge, and the additional absorption required at low energies.

The composition of the dust in DWAs may be constrained by the relative strength of the Fe I and $\mathrm{O}$ I edges, or by the detailed structure around the Fe I edge, as was done in Cyg X-1. The present MCG-6-30-15 data is consistent with silicate dust having a $\mathrm{O}: \mathrm{Fe}$ ratio of $2: 1$ or less, which could result from a process of repeated grain destruction and growth in the ISM.
The presence of pure iron grains seems unlikely given the limit on their abundance from our own Galaxy. High signal-to-noise data around the $\mathrm{O}$ I edge, or a measurement of the $\mathrm{C} I$ edge, is needed to fully exploit the observations in determining the dust composition in distant AGN.

Acknowledgements. We thank Gary Ferland for helpful discussions regarding the Cloudy models, and Julia Lee for comments on a draft of the manuscript. This research was supported by the Natural Sciences and Engineering Research Council of Canada and by the Canada Research Chair Program. This research has made use of the NASA/IPAC Extragalactic Database (NED) which is operated by the Jet Propulsion Laboratory, California Institute of Technology, under contract with the National Aeronautics and Space Administration.

\section{References}

Abolins, J. A., \& Rice, W. L. 1987, in Star Formation in Galaxies, (Washington, D.C.: NASA), 107

Ballantyne, D. R., Ross, R. R., \& Fabian, A. C. 2002, MNRAS, 336, 867

Barvainas, R. 1987, ApJ, 320, 537

Bottorff, M. C., Korista, K. T., \& Shlosman, I. 2000, ApJ, 537, 134

Brandt, W. N., Fabian, A. C., \& Pounds, K. A. 1996, MNRAS, 278, 326

Branduardi-Raymont, G., Sako, M., Kahn, S. M., et al. 2001, A\&A, 365, L140

Collinge, M. J., Brandt, W. N., Kaspi, S., et al. 2001, ApJ, 557, 2

Crenshaw, D. M., \& Kraemer, S. B., 2001, ApJ, 562, L29

Crenshaw, D. M., Kraemer, S. B., Bruhweiler, F. C., \& Ruiz, J. R. 2001, ApJ, 555, 633

Crenshaw, D. M., Kraemer, S. B., Turner, T. J., et al. 2002, ApJ, 566, 187

Crocombette, J. P., Pollak, M., Jollet, F., Thromat, N., \& Gautier-Soyer, M. 1995, Phys. Rev. B, 52, 3143

Dickey, J. M., \& Lockman, F. J. 1990, ARA\&A, 28, 215

Draine, B. T., \& Lazarian, A. 1999, ApJ, 512, 740

Elvis, M. 2000, ApJ, 545, 63

Elvis, M., Wilkes, B. J., \& Lockman, F. J. 1989, AJ, 97, 777

Fabian, A. C., Kunieda, H., Inoue, S., et al. 1994, PASJ, 46, L59

Ferland, G. J. 2002, Hazy, a Brief Introduction to Cloudy, University of Kentucky, Department of Physics and Astronomy Internal Report

Ferruit, P., Wilson, A. S., \& Mulchaey, J. 2000, ApJS, 128, 129

Fiore, F., Elvis, M., Mathur, S., Wilkes, B. J. \& McDowell, J. C. 1993, ApJ, 415, 129

George, I. M., Turner, T. J., \& Netzer, H. 1995, ApJ, 438, L67

George, I. M., Turner, T. J., Netzer, H., et al. 1998, ApJS, 114, 73

Gloter, A., Guyot, F., Martinez, I., \& Colliex, C. 2000, Am. Mineralogist, 85, 1452

Grevesse, N., \& Sauval, A. J. 1998, Space Sci. Rev., 85, 161

Halpern, J. 1984, ApJ, 281, 90

Jones, A. P., Tielens, A. G. G. M., \& Hollenbach 1996, ApJ, 469, 740

Kaastra, J. S., Mewe, R., Liedahl, D. A., Komossa, S., \& Brinkman, A. C., 2000, A\&A, 354, L83

Kaastra, J. S., Steenbrugge, K. C., Raassen, A. J. J., et al. 2002, A\&A, 386,427

Kaspi, S., Brandt, W. N., Netzer, H., et al. 2001, ApJ, 554, 216

Kaspi, S., Brandt, W. N., George, I. M., et al. 2002, ApJ, 574, 643

Komossa, S., \& Fink, H. 1997, A\&A, 327, 483

Komossa, S., \& Bade, N. 1998, A\&A, 331, L49

Kortright, J. B., \& Kim, S.-K. 2000, Phys. Rev. B, 62, 12216 
Kraemer, S. B., George, I. M., Turner, T. J., \& Crenshaw, D. M. 2000, ApJ, 535, 53

Krolik, J. H., \& Kriss, G. A. 1995, ApJ, 447, 512

Krolik, J. H., \& Kriss, G. A. 2001, ApJ, 561, 684

Laor, A., \& Draine, B. T. 1993, ApJ, 402, 441

Lee, J. C., Ogle, P. M., Canizares, C. R., et al. 2001, ApJ, 554, L13

Lee, J. C., Canizares, C. R., Fang, T., et al., 2002, in X-ray Spectroscopy of AGN with Chandra and XMM-Newton, ed. Th. Boller, S. Komossa, S. Kahn, H. Kunieda, \& L. Gallo, MPE Report, 279,9

Loyer, E., Simien, F., Michard, R., \& Prugniel, Ph. 1998, A\&A, 334, 805

Malkan, M. A., Gorjian, V., Tam, R. 1998, ApJS, 117, 25

Martin, P. G., \& Rouleau, F. 1991, in Extreme Ultraviolet Astronomy, ed. R. F. Malina, \& S. Bowyer (Oxford: Pergamon Press), 341

Mathis, J. S., Rumpl, W., \& Nordsieck, K. H. 1977, ApJ, 217, 425

Matt, G. 2000, A\&A, 355, L31

Morales, R., Fabian, A. C., \& Reynolds, C. S. 2000, MNRAS, 315, 149

Nandra, K., \& Pounds, K. A. 1992, Nature, 359, 215

Nandra, K., Fabian, A. C., George, I. M., et al. 1993, MNRAS, 260, 504

Netzer, H. 1996, ApJ, 473, 781

Orr, A., Molendi, S., Fiore, F., et al. 1997, A\&A, 324, L77
Osterbrock, D. E. 1989, Astrophysics of Gaseous Nebulae and Active Galactic Nuclei (Mill Valley, CA: Univ. Sci. Books)

Otani, C., Kii, T., Reynolds, C., et al.1996, PASJ, 48, 211

Pan, H.-C., Stewart, G. C., \& Pounds, K. 1990, MNRAS, 242, 177

Reynolds, C. S. 1997, MNRAS, 286, 513

Reynolds, C. S., \& Fabian, A. C. 1995, MNRAS, 273, 1167

Reynolds, C. S., Ward, M. J., Fabian, A. C., \& Celotti, A. 1997, MNRAS, 291, 403

Sako, M., Kahn, S. M., Branduardi-Raymont, G., et al. 2003, ApJ, in press [astro-ph/0112436]

Schulz, N. S., Cui, W., Canizares, C. R., et al. 2002, ApJ, 565, 1141

Seifert, W., \& Scorza, C. 1996, A\&A, 310, 75

Shakura, N. I., \& Sunyaev, R. A. 1973, A\&A, 24, 337

Siebert, J., Komossa, S., \& Brinkmann, W. 1999, A\&A, 351, 893

Sofia, U. J., \& Meyer, D. M. 2001, ApJ, 554, L221

Telfer, R. C., Zheng, W., Kriss, G. A., \& Davidsen, A. F. 2002, ApJ, 565,773

Tielens, A. G. G. M. 1998, ApJ, 499, 267

Turner, A. K., Fabian, A. C., Vaughan, S., \& Lee, J. C. 2003, MNRAS, in press [astro-ph/0303418]

Turner, T. J., Nandra, K., George, I. M., Fabian, A. C., \& Pounds, K. 1993, ApJ, 419, 127

Weingartner, J. C., \& Draine, B. T. 1999, ApJ, 517, 292

Yaqoob, T., McKernan, B., Kraemer, S. B., et al. 2003, ApJ, 582, 105 